\title{
1 AIM-CICs: automatic identification method for Cell-in-cell 2 structures based on convolutional neural network
}

3 Running title: Automatic identification for Cell-in-cell structures

4 Meng Tang ${ }^{1,2}$, Yan $\mathrm{Su}^{1}$, Wei Zhao ${ }^{4}$, Zubiao Niu${ }^{1}$, Banzhan Ruan ${ }^{1}$, Qinqin $\mathrm{Li}^{1,2}$, You Zheng ${ }^{1}$, 5 Chenxi Wang ${ }^{1}$, Yong Zhou ${ }^{1}$, Bo Zhang ${ }^{1,3}$, Fuxiang Zhou ${ }^{5}$, Hongyan Huang ${ }^{3 *}$, Hanping Shi ${ }^{2 *}$, 6 Qiang Sun $^{1 *}$

${ }^{1}$ Laboratory of Cell Engineering, Institute of Biotechnology; 20 Dongda Street, Beijing 100071,

9 China.

$10{ }^{2}$ Department of GI Surgery, Beijing Shijitan Hospital of Capital Medical University, 10 TIEYI 11 Road, Beijing 100038, China.

$12{ }^{3}$ Department of Oncology, Beijing Shijitan Hospital of Capital Medical University, 10 TIEYI Road, 13 Beijing 10038, China.

$14 \quad{ }^{4}$ School of Mathematical Sciences, Peking University, Beijing 100871, China.

$15{ }^{5}$ Department of Radiation and Medical Oncology, Hubei Key Laboratory of Tumor Biological 16 Behaviors, Hubei Clinical Cancer Study Center, Zhongnan Hospital, Wuhan University, Wuhan 17 430071, China.

18 These authors contributed equally: Meng Tang, Yan Su, Wei Zhao, Zubiao Niu 19

Correspondence:

Hongyan Huang

Email: hhongy1999@,126.com

Hanping Shi

Email: shihp@ccmu.edu.cn

Qiang Sun

Email: sunq@bmi.ac.cn 


\section{$31 \quad$ Abstract}

Whereas biochemical markers are available for most types of cell death, current studies on non-autonomous cell death by entosis relays strictly on the identification of cell-in-cell structure (CICs), a unique morphological readout that can only be quantified manually at present. Moreover, the manual CICs quantification is generally over-simplified as CICs counts, which represents a major hurdle against profound mechanistic investigations. In this study, we take advantage of artificial intelligence (AI) technology to develop an automatic identification method for CICs (AIM-CICs), which performs comprehensive CICs analysis in an automated and efficient way. The AIM-CICs, developed on the algorithm of convolutional neural network (CNN), can not only differentiate between CICs and non-CICs (AUC > 0.99), but also accurately categorize CICs into five subclasses based on CICs stages and cell number involved (AUC $>0.97$ for all subclasses). The application of AIM-CICs would systemically fuel researches on CICs-mediated cell death such as high-throughput screening.

Key words: cell-in-cell structure; artificial intelligence; AIM-CICs; cell death; entosis; convolutional neural network 


\section{Introduction}

Cell-in-cell structures (CICs) typically referred to the unusual eukaryotic cells involving the whole objects internalized partially or completely inside of others, which had been observed in diverse physiological and pathological samples $[1,2]$. The presence of CICs was reported to be correlated with patient prognosis in a group of human tumors, such as breast cancer [3], head and neck squamous carcinoma [4, 5], and pancreatic ductal adenocarcinoma [6]. Functional studies implicated CICs in a number of biomedical processes, including embryonic development [7], mitotic surveillance [8], tumor evolution [9], and immune homeostasis [10] and the forth. As an evolutionarily conserved process, CICs formation was underlain by multiple mechanisms, such as entosis [11], cannibalism [12] and emperitosis [13]. Among which, entosis was one of the best studied processes that generally ended up with the death of the internalized cells in an acidified lysosome-dependent way $[11,14]$. The formation of entotic CICs turned out to be a genetically controlled process, where cell internalization was driven cell-autonomously by polarized actomyosin resulted from the E-cadherin-mediated adherens junctions $[15,16]$, and coordinated by a mechanical ring interfacing in between them [17]. Additionally, an ever-expanding set of factors, acting through either actomyosin, or adherent junctions or mechanical ring, were identified as important regulators [18, 19, 20,21].

Despite great progress made over the past decade, the studies on CICs formation were, however, based on the over-simplified readout of CICs counts that was performed manually, which is not only labor-intensive and time-consuming, but also sharply incompatible with the complex CICs formation per se. First, since CICs formation is a dynamic process preceding 
through sequential steps including cell-cell contact, penetration and closing [22], therefore, it generally gives rise to CICs at different stages displaying morphologies of partial or complete. Second, the CICs morphologies were further complicated by the involvement of multiple cells, which frequently resulted in structures of "cell-in-cell-in-cell" or even more. Third, due to personal experiences and preferences, the CICs judgment and inclusion-exclusion criteria for analysis varied from investigators to investigators, making it hard to compare across studies from different labs, or even studies from different investigators in one lab. In addition, manual quantification is rather inefficient in dealing with a large number of samples that may serve the screening purpose. Thus, the traditional CICs quantification reported results of less informative, hardly comparable and low-throughput, which calls for more efficient and informative ways for the quantification of CICs.

Recent years had witnessed the rapid development of image-based artificial intelligence (AI) technology in assisting biomedical practices. For example, by using a single convolutional neural networks (CNN) algorithm, Esteva et al demonstrated the classification of skin lesions in performance on par with all tested experts [23]. Lin et al developed a ResNeXt WSL model that achieved impressive performance (94.09\% accuracy, 92.79\% sensitivity, and $98.03 \%$ specificity) in making chromosome cluster type identification [24]. Actually, simply based on microscopic images, AI algorithms were quite competent in analyzing most, if not all, biological events such as the early onset of pluripotent stem cell differentiation [25], tumor cell malignancy [26], mitosis staging [27], and the like. The remarkable potentials in accuracy and efficiency make AI-based image analysis an ideal method for comprehensive and reliable CICs quantification.

In this study, based on RGB fluorescent microscopic images, we employed the deep CNN 
algorithms (Faster-RCNN and ResNet) to evaluate a large amount of cell candidates with defined subtypes and trained a multiclassfier for the recognition of subdivided CICs, which was named as AIM-CICs abbreviated from Automatic Identification Method of Cell-In-Cell structures. The AIM-CICs exhibited a high level of sensitivity and specificity, as evidenced by AUC values of $>$ 0.97 for all tasks, in differentiating CICs from non-CICs, and identifying subtyped CICs from multiple cells. The development and application of AIM-CICs hold the promise of speeding up CICs-related studies, such as deciphering the molecular controls of CICs formation in a finer resolution, and enabling image-based systemic screening by high-content microscopy.

\section{Results}

\section{The deep-learning framework of AIM-CICs}

In this work, we conducted a framework of object detection and classification based on manual annotation in the training and validation set, and then performed inspections in the test set (Fig. 1). For an RGB-format image, the proposed system performs two consecutive steps. First, a Faster-RCNN [28] network with ResNet-50 [29] backbone was formulated to find the cell regions and extract the candidate patches. Second, each candidate, representing one cell or CICs, was classified by an ResNet-101 network based on the cellular morphology. Subsequently, those subdivided candidates of the predicted results were grouped into different folders, and marked out on the original locations of the corresponding images.

\section{Cell region detection and extraction}

Cell region detection is the initial task to investigate microscopic images. According to the basic cell components, we acquired the fluorescent microscopic images with red channel for membrane 
113 and blue channel for nucleus. Along with the bright field, the merged images could be further

114 composited into RGB format with variant cell quantities and brightness values (Fig. S1a). The

115 extraction of cell candidate aims to propose regions of interest (ROI) that potentially involved CIC

116 structures. This step served to reduce the searching space and improve efficiency of subsequent

117 steps in a high-content study. Initially, four pieces of MCF7 images and four pieces of MCF10A

118 images, which included 2164 cells in total, were used as the training set for cell region detection.

119 Through manually annotating these images using VGG Image Annotator (VIA,

120 https://www.robots.ox.ac.uk/ vgg/software/via/) (Fig. 2a), cell region detection was further treated

121 as a classic 1-class object detection task through the Faster-RCNN [28] network with ResNet-50

122 [29] backbone. Specifically, during training, we have performed random flip, random rotation and

123 random scale for data augmentation, which greatly expanded the data's diversity. Following the

124 training process (Fig. 2b), we ensured the applicability of this step with average of 0.88 precision

125 and 0.96 recall (IoU 0.1) by randomly testing on 10 pieces of MCF7 and MCF10A images, which

126 covered 2398 cells (Fig. 2c).

127 It is believed that factors, such as cell morphology, sample density, as well as image brightness, do

128 impact the accuracy of target detection and recognition. In the data collected this study, MCF10A

129 samples generally displayed a larger cell size and much more complicated pattern of CICs as

130 compared with MCF7 samples (Fig. S1a). Based on the precisely manual labeling, we could

131 minimize the effect of target varieties among MCF7 and MCF10A samples (Fig. S1b), except for

132 the over-exposed fluorescent images that should be excluded in the processing of the primary

133 images. Eventually, we exported the patches of detected cell regions of the entire RGB-format images for the following analysis. 


\section{Definition of the structural subtypes of CICs}

To classify the cell-in-cell structures, we first divided the traditional CICs into five structural subtypes, including (a) partial, with more than $30 \%$ of the internalizing cells were enclosed, but not fully, by the outer cells; (b) one-in-one, with only one cell fully internalized, (c) two-in-one, with two cells were fully internalized; (d) in turn, a nested CICs with multiple cells sequentially internalized into neighboring cells; (e) complicated, a complex CICs generated by four or more cells (Fig. 3a). Considering the potential complexity, two kinds of breast cell lines including MCF7 and MCF10A were investigated, in which the total rate of CICs and its subtypes showed great discrepancy according to the manually labeling (Fig. 3b). In total, 17 pieces of MCF7 images and 85 pieces of MCF10A images were enrolled in this study, the cell number of each image ranged from 100 to 600 , and from 30 to 200 , respectively (Fig. 3c). The overall CICs rate of each image counted from $1 \%$ to $85 \%$ (Fig. 3d).

\section{Multi-Subtype classification achieved by the AIM-CICs}

The obtained cell candidates were used to train ResNet101 model for the purpose of CICs recognition (Fig. S2a). Practically, we used 13 pieces of MCF7 images and 32 pieces of MCF10A images as the training set, which had 4026 MCF7 cells with a CICs rate of $11 \%$ and 3912 MCF10A cells with a CICs rate of $32 \%$ (Fig. S2b). Based on the morphological features of cell candidates, five subtypes of CICs were manually labeled for each cell candidate in the training and validation set. The distribution of each subtype of CICs showed remarkable discrepancy, as well as in the test set (Fig. 4a-b). To improve the practicality of the model, we defined a F-category from the non-CIC candidates. The F-category contains ambiguous structures that were hard to tell their identities by both experienced experts and AI algorithm, therefore, were generally removed 
from the sample counting (Fig S2c-d).

As shown in Fig. 4c, data training progressively increased the prediction accuracy to a considerable level for each subtype. In both training and validation sets, the comprehensive accuracy of integrated CICs (involving a, b, c, d, e types) and non-CIC type (including F category) revealed approving performance (Fig. 4d). Moreover, the AIM-CICs also exhibited impressive performance as indicated by the AUC of more than 0.97 for each CICs subtype (partial 0.9761, one-in-one 0.9807, two-in-one 0.9872, in turn 0.9709, complicated 0.9984) (Fig. 4e-f) in the test set. Additionally, for the low-quality images in the test set that displayed unclear cell regions and were eventually removed for further analysis, their recognition also reached an ideal AUC of 0.99 (Fig. S2e). Together, the AIM-CICs performed accurate recognition of CICs on independent datasets of MCF10A and MCF7 cells, suggesting the generalizability of this model.

\section{Visualization of morphological features and output}

To better understand what the model learnt from the annotated data, we extract features from the output of network's global average pooling layer and applied t-SNE to reduce dimension to 2D for visualization. For the training set, each group of cell samples represented independent clusters, except for cell candidates in the circled region (Fig. 5a). Backtracking the training data identified that these were candidates categorized into two subtypes due to erroneous manual annotation. Thus, the t-SNE-based clustering would be a visualized way for error-correction in recognizing CICs. For the test set (Fig. 5b), subtypes of CICs were clustered into close, but clearly distinct, regions, whereas F-category was neighboring to the area of non-CIC as expected. Moreover, following the comprehensive recognition under a specified confidence threshold, we were able to accurately locate each structure with a predicted value on the original images (Fig. 5c). 
179

\section{Application of AIM-CICs in an experimental setup}

To explore the potential implication of AI-based recognition of CICs in a biological context, we included a functional experiment as an example of subtype profiling. In this analysis, the confidence threshold was set to 0.2 for more informative identification (Fig. 6a). As the results showed, though all of the three truncations of ARHGAP36, a molecule identified to be a regulator of CICs formation in a screening study [20], resulted in impaired formation of CICs, the alterations of CICs subtypes were rather different (Fig. 6b-c). While the truncated GAP36 (1-194) had little impact on the formation of partial CICs (Fig. 6b-d), the majority of CICs were in completed form (including all CICs subtypes except for the partial) in cells expressing the truncated GAP (118-194) or GAP (195-395) (Fig. 6b-c), suggesting that the N-terminal region (1-117) of ARHGAP36 might function to slow down the process of cell internalization. Meanwhile, the C-terminal region of ARHGAP36 was likely to be responsible for the closing step of CICs formation as evidenced by comparable formation of completed CICs between control and GAP (195-395)-expressing cells (Fig. 6b and 6e-h). Moreover, the GAP (118-194) seemed to be the major region that drives cell internalization as it promoted the formation of completed CICs at a rate comparable to the GAP (1-194) region. Furthermore, though the N-terminal region might negatively regulate the speed of CICs formation, it did function positively to promote cell internalization as its truncation significantly reduced the formation of both partial and total CICs (Fig. 6b-d). Thus, the AIM-CICs algorithm allows us, for the first time, to accurately dissect the impacts of different domains or molecules on CICs formation in a heretofore underappreciated resolution.

\section{Discussion}



accuracy and potentially featured insights, we, for the first time, explored the application of images accumulated from previous studies, we developed the AI-based identification algorithm AIM-CICs, which was trained with distinct illumination, textures, and density, in order to deliver an optimal performance in cell region detection and multiple subtypes classification, despite of the unseen perturbations.

In the proposed system, we set up two tasks, of which, a classic 1-class object detection model was formulated to find cell regions as the first task, followed by multi-class object recognition as the second task. Comparing with the traditional end-to-end manner, i.e., to train a multi-class detection model with different kinds of cells marked simultaneously, our model of separated detection will achieve the flexibility for the raw samples to be recategorized and repurposed. In AIM-CICs developed in this study, the second task included a well-trained 7-category classifier (5 structures, which is compatible with the cell candidates from the first step. This two-step algorithm is also advantageous in debugging the possible mechanisms leading to inferior final algorithm may fall short of efficiency (speed) as compared to the end-to-end multi-class detection model which could utilize a shared feature extraction backbone. 
accomplished with the involvement of at least two cells, but not one cell in other programs like apoptosis, necrosis and the forth [30]. Therefore, mechanistic study is a challenging task for the field of CICs-mediated death, which was further complicated by the fact of lacking a reliable biochemical marker. Current studies on CICs relayed on the morphology-based binary quantification, that is, CICs or non-CICs. Here, CICs were usually defined as structures with more than $1 / 2$, or $2 / 3$ in some studies, of the inner cell body being internalized/enclosed by the outer cell. This oversimplified quantification of CICs, did move the field forward over the past decade, however, provided rather coarse information over a more complicated process [22, 31]. CICs formation is a stepwise process that could be empirically subdivided into three major stages: 1) the early initiation stage from cell-cell contact to about $1 / 3$ of the inner cell body being internalized, this stage was primarily driven by cell-cell adhesion and assisted by cytoskeleton remodeling; 2) the middle internalization stage covering the whole process of cell internalization that was primarily driven by active actomyosin contraction within the inner cells, and coordinately assisted by the outer cells; 3 ) the final closing stage that may involve in tail cutting and membrane fusion, this a process rarely being investigated largely because it is technically challenging. Furthermore, CICs formation is a dynamic process that may have multiple cells, either sequentially or simultaneously, form a complicated structure that may contain more than one cell inside (Fig. 3a). The regulation of this feature is completely unknown for the field yet, but might be conceptually feasible as it was reported in phagocytosis that the number of corpses engulfed by a phagocyte was genetically controlled [32]. Taking these two factors (stage and cell number) into account will produce an even higher dimensional complexity, which, however, was missed from the traditional analysis by the binary quantification. The implantation of AIM-CICs enables us to make a more 
sophisticated description of CICs phenotypes, which would help identify finer molecular control.

For example, though expression of the GAP (195-395) domain did not influence the frequency of simple CICs, where only one cell was enclosed (one-in-one in Fig. 6e), it did result in significantly reduced formation of complex CICs, where more cells were enclosed by one outer cell may facilitate the internalization of multiple cells to form complex CICs, which warrants further

251 functional validation.

In addition to mechanistic investigation, AIM-CICs is also promising in enabling high-content based screening for therapeutic compounds that target CICs formation considering their pivotal roles in multiple biomedical processes such as cancer [1]. While high throughput screening generally relies critically on a reliable biochemical marker that is currently unavailable to CICs formation, the related systemic screening, which would be labor-intensive and time-consuming if worked out by manual annotation, had yet been reported. Empowered with AIM-CICs and high-content microscopy, the systemic screening would be feasible in the near future.

\section{Acknowledgements}

260 We thank Dr Lulin Zhou, Xiaoyi Jiang, He Ren, Yichao Zhu, Yuqi Wang, Lihua Gao, Zhaolie

261 Chen, and the members of the Sun lab for the constructive discussions and assisting manual 262 labeling.

\section{Funding statement}

264 This work was supported by Beijing Municipal Natural Science Foundation (KZ202110025029 to 
Science Foundation of Beijing Shijitan Hospital of CMU (No. 2019-q02 to MT).

\section{Author contributions}

Concept and design: QS; CNN Model Training and Analysis: WZ; Data collection: MT, ZBN,

CXW, and BZR; Data interpretation: QS, WZ and MT; Figures: MT, YS, WZ and QS; Manuscript: manuscript.

\section{Declaration of interests}

275 The authors declare no competing interests.

\section{Ethics statement}

277 This study did not involve ethical approval.

\section{Materials and methods}

\section{Image processing and softwares}

An entire dataset involving 17 pieces of MCF7 images and 85 pieces of MCF10A images were

281 obtained from Sun's lab. As detailed protocol described [33], the fluorescently labeled cells were necessary to be stained with discrepant colors for each cell components, such as, red for cytomembrane (E-Cadherin, 1:200, BD Biosciences, 610181) with secondary antibodies Alexa Fluor 568 anti-rabbit (1:500; Invitrogen; A11036), and blue for cytoblast (DAPI, Sigma D8417). 
microscopy (Nikon Ti-E microscope, Nikon NIS-Elements AR 4.5 software), along with bright color for the background. For algorithm performing, each sample with three single-channel images was transformed into an RGB format with value rescaled to 0 - 255. Softwares used and algorithms developed in this study include: Python (http://www.python.org./); PyTorch Detectron2 (https://github.com/facebookresearch/detectron2).

\section{Cell region labeling and candidates extraction}

294 After acquiring the processed images, we manually annotated the cell regions through VGG Image Annotator (https://www.robots.ox.ac.uk/ vgg/software/via/). Based on the annotated images, a classic 1-class object detection task was carried out for cellular morphological learning. The model we used is a Faster-RCNN [28] network with ResNet-50 [29] backbone. Since the original resolution of microscopic image is $2160 \times 2560$ which is too large for Faster-RCNN training, we first split each image into $4 \times 4$ grids, then follow the common practice to train the model. For data augmentation, we use random flip, random rotation, and random scale to expand diversity of data. optimizer with momentum 0.9. As the output of the Faster-RCNN network, the patches of detected cell regions were exported as candidate sequences for further steps.

\section{Manual classifications of cell-in-cell structures}

The manual definition of cell-in-cell structural classification primarily included bipartite-class, 
with more than $30 \%$ of the internalizing cells were enclosed, but not fully, by the outer cells; (b) one-in-one, with only one cell fully internalized, (c) two-in-one, with two cells were fully internalized; (d) in turn, a nested CICs with multiple cells sequentially internalized into neighboring cells; (e) complicated, a complex CICs generated by four or more cells. To refine the output results, we added a F-category among non-CICs, which was defined as unclear or not sure for the cell recognition and needs to be removed for the quantitative analysis. The cell candidates involved in the training set were verified together by an expert group consisted of 6 members in the lab.

\section{Multiple classification model}

We used the ResNet101 model as our classifier and the input size was set to 224. Since this model could take the detection model's output as input, we cropped cell samples using detection model and manually labeled them with corresponding cell types. During training, each sample was first padded to square and then resize to $224 \times 224$. Both horizontal and vertical random flip were performed. We trained our model for 250 epochs with batch size of 32, using SGD optimizer with learning rate of 0.001 and momentum of 0.9 . To alleviate overfitting, a dropout layer with $p=0.25$ was set right before feature went into the final fully connected layers. To choose hyper-params, we kept $20 \%$ samples as validation set. Eventually, the prediction results could be visualized on the folders of each cell type. Importantly, when applying our model for inference, the test samples should be pad and resize in the same way as training. Our model is a 7 classes classifier, and it outputs a 7 elements vector 
representing the probability for the test sample to belong to each type. Traditionally, the predicted type should be the type with maximum probabilities. In practice, to increase precision, we predict cells that have predicted probability lower than 0.2 as non-CIC, even if the non-CIC probability is not the maximum for it. For example, if the predicted output is $[0.1,0.18,0.12,0.15,0.15,0.13,0.17]$ output the classifications into specific folders of each cell type, and obtain the visualized results that marked with individual colors on the original image.

\section{Performance analysis of detection model}

In deep learning community, the most common metric used for quantitatively comparing detection models' performance is mean average precision (mAP), as proposed in [34] and [35]. However, since our work mainly focused on multi-type CICs classification instead of general object detection technique, we reported our detection result in a more practical recall/precision manner. In detail, we kept detection model's output instances with confidence $>0.1$ as model's prediction and calculate metrics at two different Intersection over Union (IoU) thresholds $0.5 / 0.1$. Under requires only loosely overlapping.

\section{Features visualization}

To better understand what the classification model learns from labeled samples, we extracted features from each cell sample and visualize them in a 2D space. The feature we used is the output of network's global average pooling layer, which is right before the final classification layer. This 
352 2048D feature is the deepest and the most semantic so it can represent the information extracted

353 by the network from a corresponding input image. To visualize these 2048D features, we uses the

354 t-SNE method for dimensionality reduction to transform each feature to $2 \mathrm{D}$ [36]. t-SNE is a

355 popular method for visualizing high-dimension data since it can keep most of the original data

356 structure during dimensionality reduction.

\section{Evaluation criteria for classification models}

359 The output of classification model was evaluated by the universal criteria, such as, sensitivity (Se

360 or recall), specificity ( $\mathrm{Sp}$ ), precision, the receiver operating characteristic (ROC) curve, and the

361 area under ROC curve (AUC). The equations were listed as follows:

$362 \quad(1) \mathrm{Se}($ recall $)=\mathrm{TP} /(\mathrm{TP}+\mathrm{FN})$

363 (2) $\mathrm{Sp}=\mathrm{TN} /(\mathrm{TN}+\mathrm{FP})$

364 (3) Precision $=\mathrm{TP} /(\mathrm{TP}+\mathrm{FP})$

365 True positive (TP) stands for the accounts of positive CICs which are correctly recognized as

366 positive CICs. False positive (FP) stands for the number of negative CICs that are incorrectly

367 recognized as positive CICs. False negative (FN) stands for the accounts of positive CICs which

368 are incorrectly recognized as negative CICs. True negative (TN) stands for the number of negative

369 CICs correctly recognized as negative CICs.

\section{Statistical analysis}


bioRxiv preprint doi: https://doi.org/10.1101/2021.02.26.432996; this version posted February 27, 2021. The copyright holder for this preprint (which was not certified by peer review) is the author/funder, who has granted bioRxiv a license to display the preprint in perpetuity. It is made available under aCC-BY-NC-ND 4.0 International license.

372 Categorical data are expressed as frequencies (\%) and were tested by a two-tailed Student's t-test.

373 P values were calculated by Excel or GraphPad Prism software. The level of significance was set

374 at $p<0.05$.

375

376

377 


\section{References}

1. Fais S, Overholtzer M. Cell-in-cell phenomena in cancer. Nat Rev Cancer 2018, 18(12): 758-766.

381 2. Wang X. Cell-in-cell phenomenon: A New Paradigm in Life Sciences. Current molecular medicine 2015, 15(9): 810-818.

3. Zhang X, Niu Z, Qin H, Fan J, Wang M, Zhang B, et al. Subtype-Based Prognostic Analysis of Cell-in-Cell Structures in Early Breast Cancer. Front Oncol 2019, 9: 1-12.

4. Schwegler M, Wirsing AM, Schenker HM, Ott L, Ries JM, Buttner-Herold M, et al. Prognostic Value of Homotypic Cell Internalization by Nonprofessional Phagocytic Cancer Cells. BioMed research international 2015, 2015: 359392.

5. Fan J, Fang Q, Yang Y, Cui M, Zhao M, Qi J, et al. Role of Heterotypic Neutrophil-in-Tumor Structure in the Prognosis of Patients With Buccal Mucosa Squamous Cell Carcinoma. Front Oncol 2020, 10(2284): 541878.

6. Huang $\mathrm{H}, \mathrm{He} \mathrm{M}$, Zhang $\mathrm{Y}$, Zhang $\mathrm{B}$, Niu Z, Zheng $\mathrm{Y}$, et al. Identification and validation of

7. Lee Y, Hamann JC, Pellegrino M, Durgan J, Domart MC, Collinson LM, et al. Entosis Controls a

8. Liang J, Niu Z, Zhang B, Yu X, Zheng Y, Wang C, et al. p53-dependent elimination of aneuploid

9. Sun $Q$, Luo $T$, Ren $Y$, Florey $O$, Shirasawa $S$, Sasazuki $T$, et al. Competition between human

10. Davies SP, Reynolds GM, Wilkinson AL, Li X, Rose R, Leekha M, et al. Hepatocytes Delete

11. Overholtzer M, Mailleux AA, Mouneimne G, Normand G, Schnitt SJ, King RW, et al. A nonapoptotic cell death process, entosis, that occurs by cell-in-cell invasion. Cell 2007, 131(5): 966-979.

14. Su Y, Ren $H$, Tang $M$, Zheng $Y$, Zhang B, Wang $C$, et al. Role and dynamics of vacuolar $\mathrm{pH}$

15. Sun $Q$, Cibas ES, Huang $H$, Hodgson L, Overholtzer $M$. Induction of entosis by epithelial cadherin expression. Cell Res 2014, 24(11): 1288-1298. 
18. Wang C, Chen A, Ruan B, Niu Z, Su Y, Qin H, et al. PCDH7 Inhibits the Formation of Homotypic Cell-in-Cell Structure. Front Cell Dev Biol 2020, 8(329): 1-12.

19. Liang J, Fan J, Wang M, Niu Z, Zhang Z, Yuan L, et al. CDKN2A inhibits formation of homotypic cell-in-cell structures. Oncogenesis 2018, 7(6): 1-8.

20. Ruan B, Wang C, Chen A, Liang J, Niu Z, Zheng Y, et al. Expression profiling identified IL-8 as a regulator of homotypic cell-in-cell formation. BMB Rep 2018, 51(8): 412-417.

21. Hinojosa LS, Holst M, Baarlink C, Grosse R. MRTF transcription and Ezrin-dependent plasma membrane blebbing are required for entotic invasion. J Cell Biol 2017, 216(10): 3087-3095.

22. Niu Z, He M, Sun Q. Molecular mechanisms underlying cell-in-cell formation: Core machineries and beyond. J Mol Cell Biol 2021.

23. Esteva A, Kuprel B, Novoa RA, Ko J, Swetter SM, Blau HM, et al. Dermatologist-level classification of skin cancer with deep neural networks. Nature 2017, 542(7639): 115-118.

24. Lin C, Zhao G, Yin A, Yang Z, Guo L, Chen H, et al. A novel chromosome cluster types identification method using ResNeXt WSL model. Med Image Anal 2020, 69: 101943.

25. Waisman A, La Greca A, Mobbs AM, Scarafia MA, Santin Velazque NL, Neiman G, et al. Deep Learning Neural Networks Highly Predict Very Early Onset of Pluripotent Stem Cell Differentiation. Stem Cell Reports 2019, 12(4): 845-859.

26. Oei RW, Hou G, Liu F, Zhong J, Zhang J, An Z, et al. Convolutional neural network for cell classification using microscope images of intracellular actin networks. PLoS One 2019, 14(3): e0213626.

27. Mao Y, Han L, Yin Z. Cell mitosis event analysis in phase contrast microscopy images using deep learning. Med Image Anal 2019, 57: 32-43.

28. Ren S, He K, Girshick R, Sun J. Faster r-cnn: towards real-time object detection with region proposal networks. IEEE Transactions on Pattern Analysis and Machine Intelligence 2015, 39(6): In: NIPS. (2015).

29. He K, Zhang X, Ren S, Sun J. Deep Residual Learning for Image Recognition. IEEE Conference on Computer Vision \& Pattern Recognition 2016: In: CVPR. (2016).

30. Galluzzi L, Vitale I, Aaronson SA, Abrams JM, Adam D, Agostinis P, et al. Molecular mechanisms of cell death: recommendations of the Nomenclature Committee on Cell Death 2018. Cell Death Differ 2018, 25(3): 486-541.

31. Rizzotto D, Villunger A. P53 clears aneuploid cells by entosis. Cell Death Differ 2020, 10.1038/s41418-020-00659-x.

32. Park D, Han CZ, Elliott MR, Kinchen JM, Trampont PC, Das S, et al. Continued clearance of apoptotic cells critically depends on the phagocyte Ucp2 protein. Nature 2011, 477(7363): 220-224.

33. Sun Q, Overholtzer M. Methods for the study of entosis. Methods Mol Biol 2013, 1004: 59-66.

34. Everingham M, Van Gool L, Williams CKI, Winn J, Zisserman A. The Pascal Visual Object Classes (VOC) Challenge. International Journal of Computer Vision 2009, 88(2): 303-338.

35. Lin TY, Maire M, Belongie S, Hays J, Zitnick CL. Microsoft COCO: Common Objects in Context. European Conference on Computer Vision 2014: In: ECCV. (2014).

36. Maaten. Lvd, G. H. Visualizing data using t-SNE. Journal of Machine Learning Research 2008, 9: 2579-2605. 
Fig. 1 The workflow for the development of AIM-CICs.

Fig. 2 Cell regions detection and extraction. a Using VGG Image Annotator to annotate cell regions Intersection over Union.

Fig. 3 Image processing and cell candidate regions. a Representative images of five CICs subtypes. are listed in supplementary Fig. S1a. b Percentage of different CICs subtypes for MCF7 and MCF10A cells. c The number of cell candidates extracted from each MCF7 and MCF10A image. Samples contained 17 pieces of MCF7 images and 85 pieces of MCF10A images. The columns in orange and purple represent for samples used in the training set. $\mathbf{d}$ The count and frequency of CICs for individual MCF7 or MCF10A image. 
respectively. In the MCF10A samples, the CIC counts were 1269 (a) and 1948 (b), respectively.

Supplementary figures associated: Supplementary Fig. S2. c The prediction accuracy of AIM-CICS for each subtype in a 250 epochs of learning process. d The integrated accuracy of AIM-CICS for CICs and non-CIC type in the training and validation set in a 250 epochs of learning process. CICs included non-CIC. e Representative images of each CICs subtype predicted in the training set. $\mathbf{f}$ The ROC curves for each CICs subtype in the test set.

Fig. 5 Visualization of sample features and output. a,b The two-dimension visualization of CICs subtypes in the training set (a) and the test set (b). The samples circled out were those predicted by the AIM-CICs to be miscategorized manually. c A representative image showing the recognition result of were marked on the up-left corner of each structure. The structures in the right were cropped from the image in the left.

Fig. 6 Analysis of CICs subtypes in an experimental setup by AIM-CICs. a The visualized recognition results of AIM-CICs in an experiment, where MCF10A cells expressed the empty vector (control) and three truncated mutants of ARHGAP36 (1-194, 118-194, 195-395), respectively. b,c expressing different ARHGAP36 mutants. ( $\mathrm{n}=934$ cells for control, 1060 cells for 1-194, 1392 cells for 118-194, 852 cells for 195-395). d-h The frequencies of subtyped CICs in MCF10A cells 
bioRxiv preprint doi: https://doi org/10.1101/2021.02.26.432996; this version posted February 27, 2021. The copyright holder for this preprint (which was not certified by peer review) is the author/funder, who has granted bioRxiv a license to display the preprint in perpetuity. It is made available under aCC-BY-NC-ND 4.0 International license.

data points. * for $p<0.05, * *$ for $p<0.01, * * *$ for $p<0.001$ (two-tailed Student's t test).

Fig. S1 Format transformation and cell region detection. a Representative images of different model. Each cell region was indicated by a box with a predicted confidence score.

Fig. S2 Binary subtyping by the AIM-CICs. a Representative images for CICs and non-CICs. b The

\section{Figure 1}

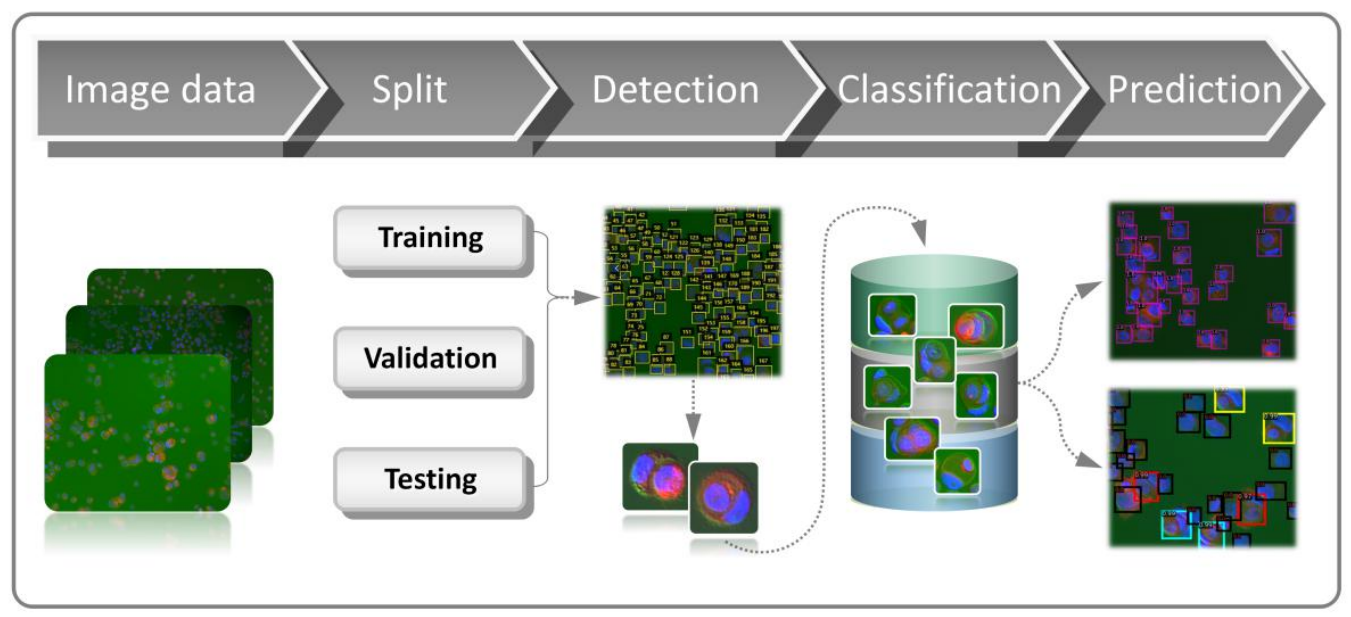


bioRxiv preprint doi: https://doi org/10.1101/2021.02 26.432996; this version posted February 27, 2021. The copyright holder for this preprint (which was not certified by peer review) is the author/funder, who has granted bioRxiv a license to display the preprint in perpetuity. It is made available under aCC-BY-NC-ND 4.0 International license.

\section{Figure 2}
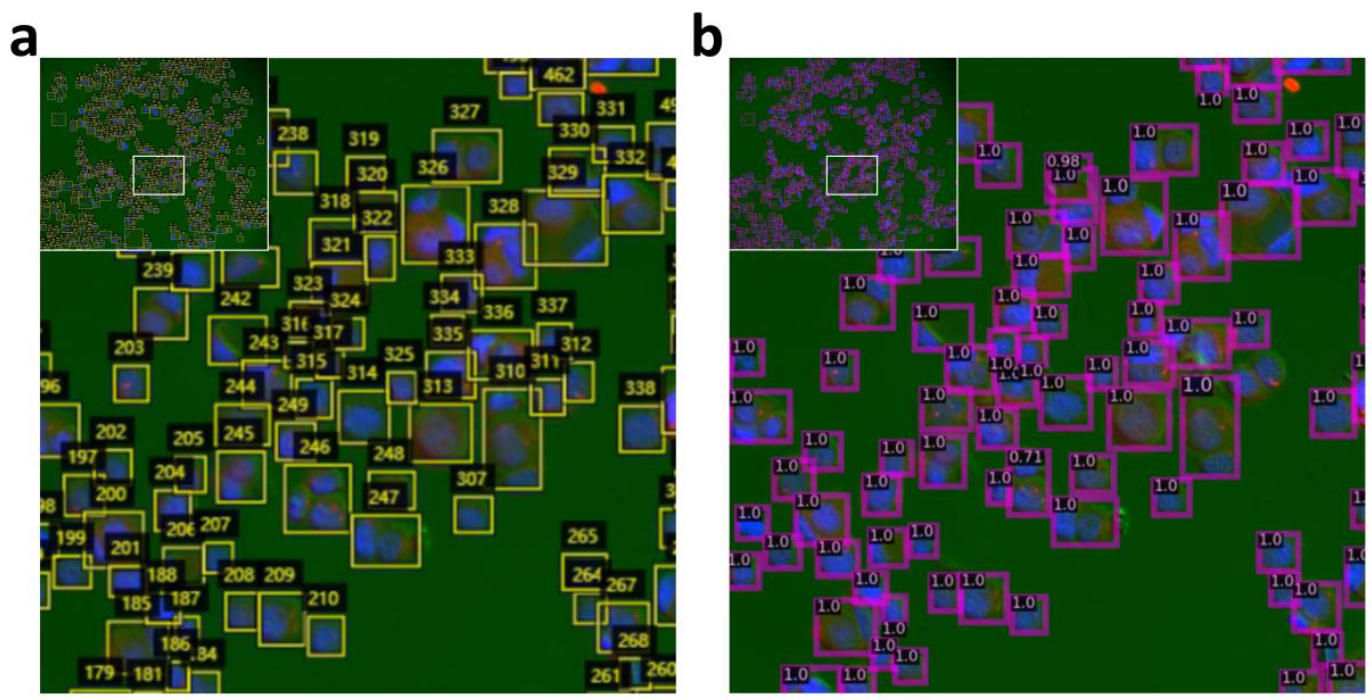

C

\begin{tabular}{lcccccccc}
\hline \multicolumn{1}{c}{ Group } & N_GT & N_prediction & N_RECALL & $\begin{array}{c}\text { IoU } 0.1 \\
\text { RECALL }\end{array}$ & PRECISION & N_RECALL & RECALL & PRECISION \\
validation_MCF7 & 1783 & 1782 & 1691 & 0.947 & 0.947 & 1650 & 0.923 & 0.923 \\
validation_MCF10A & 347 & 367 & 336 & 0.968 & 0.904 & 333 & 0.960 & 0.897 \\
testing & 268 & 331 & 258 & 0.962 & 0.781 & 238 & 0.893 & 0.725 \\
\hline
\end{tabular}


bioRxiv preprint doi: https:/doi.org/10.1101/2021.02 26.432996; this version posted February 27, 2021. The copyright holder for this preprint (which was not certified by peer review) is the author/funder, who has granted bioRxiv a license to display the preprint in perpetuity. It is made available under aCC-BY-NC-ND 4.0 International license.

\section{Figure 3}

a

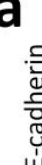

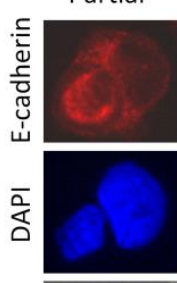

늠
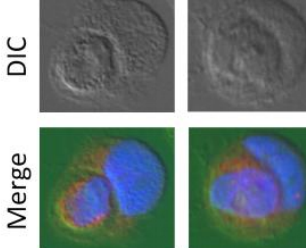

Cell-in-cell structure subtypes

c

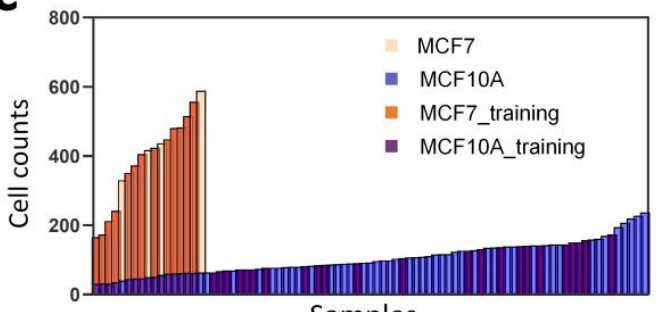

Samples
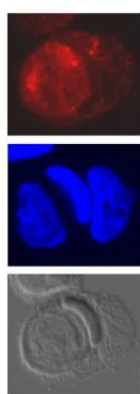

b

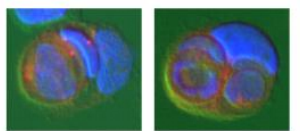

d

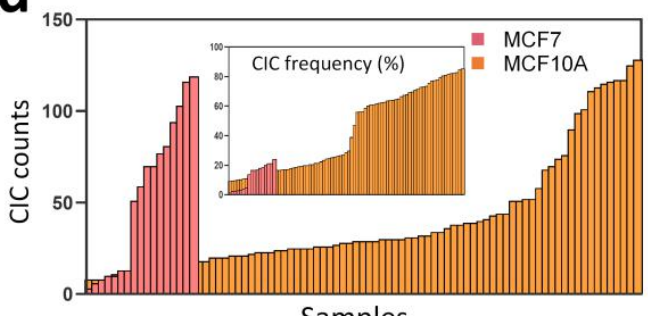

Samples 
bioRxiv preprint doi: https://doi.org/10.1101/2021.02.26.432996; this version posted February 27, 2021. The copyright holder for this preprint (which was not certified by peer review) is the author/funder, who has granted bioRxiv a license to display the preprint in perpetuity. It is made available under aCC-BY-NC-ND 4.0 International license.

\section{Figure 4}

a

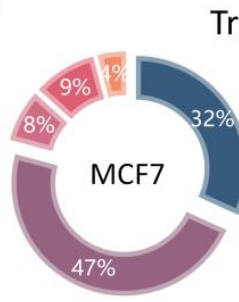

a_partial

C
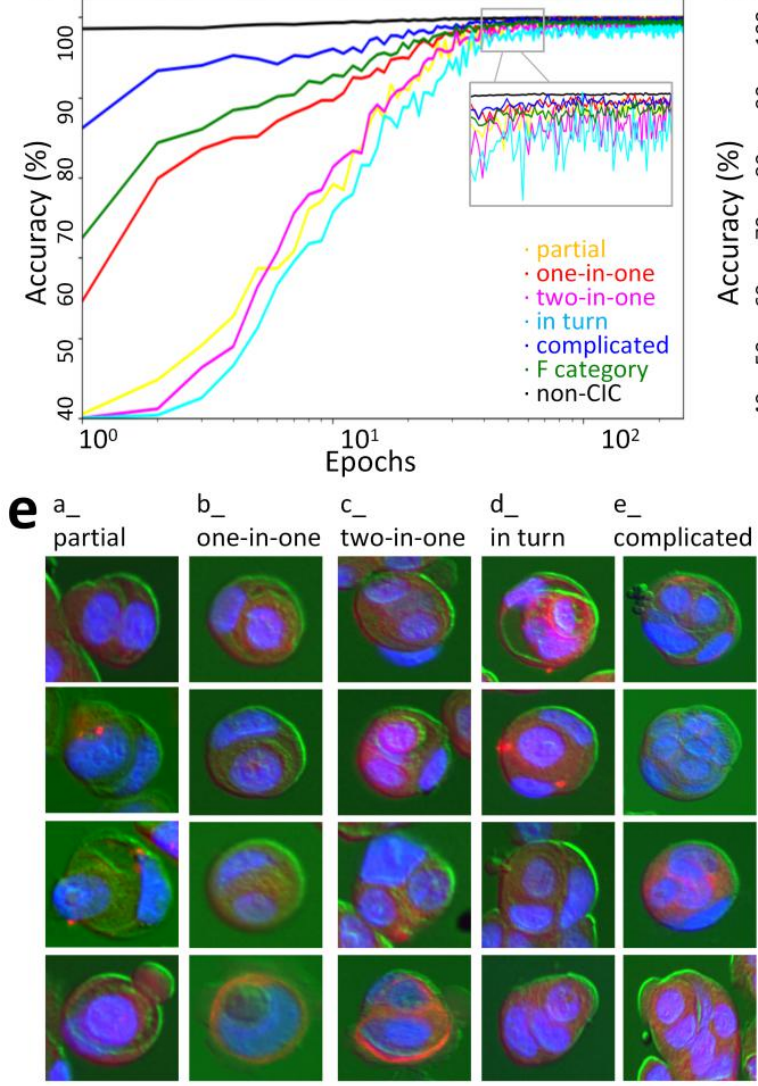

b

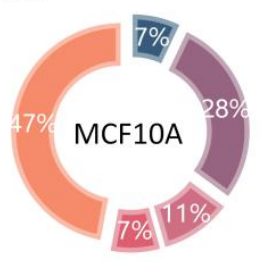

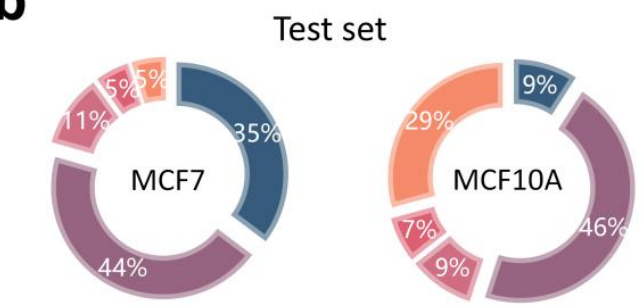

Test set

e_complicated

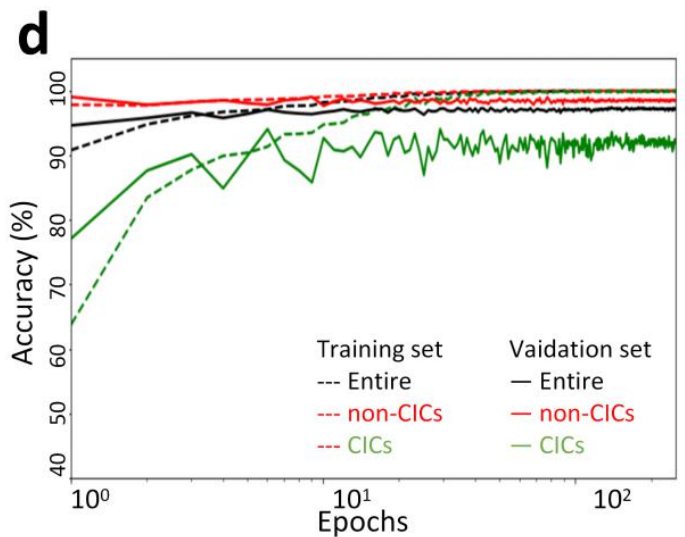

f

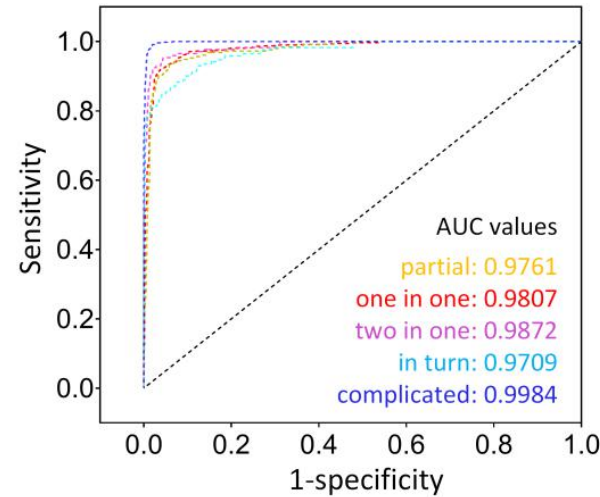


bioRxiv preprint doi: https://doi org/10.1101/2021.02 26.432996; this version posted February 27, 2021. The copyright holder for this

\section{Figure 5}

a

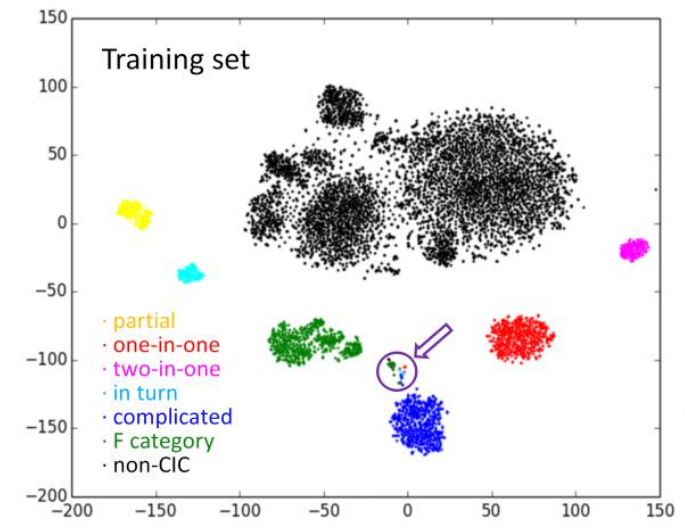

C

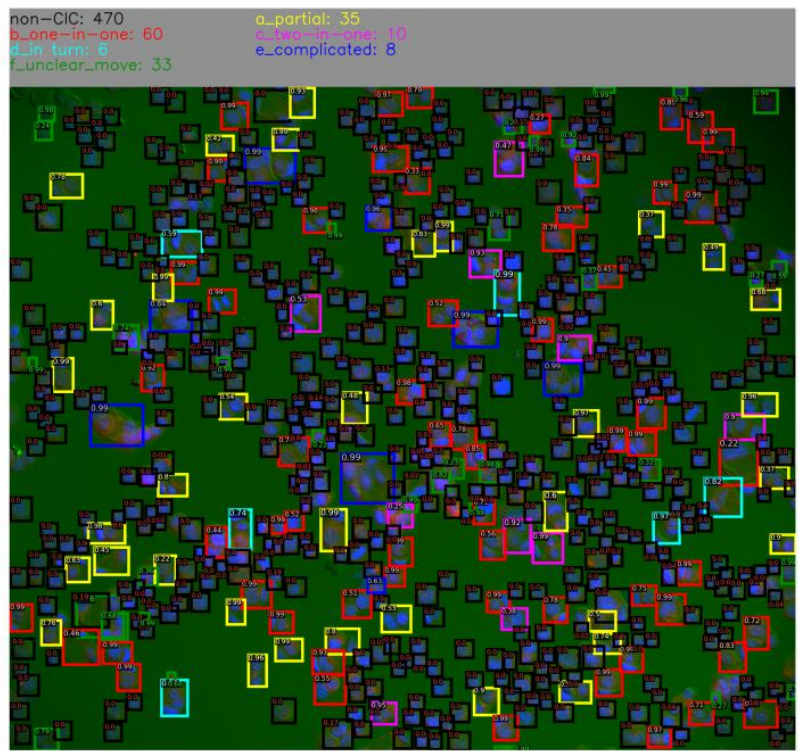

b

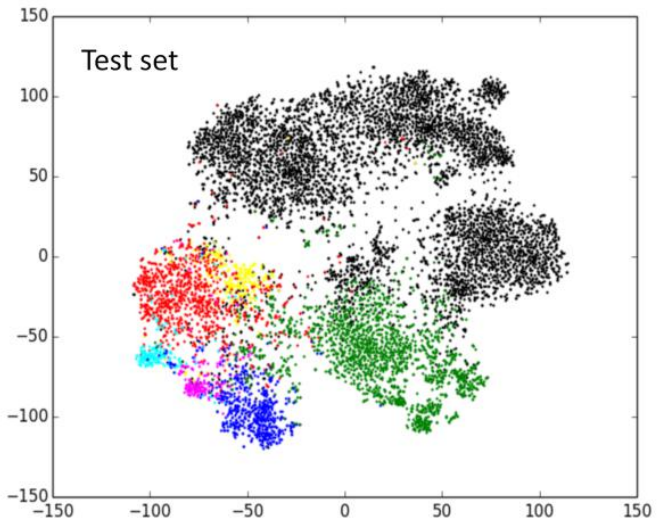

.
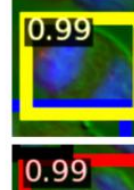

ธิ 0.9

蓄

ธ 1

0.93

¿े

לุ.

$\stackrel{5}{\subseteq}$

0

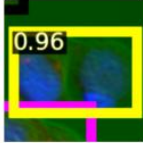

0.54

0.99

0.52

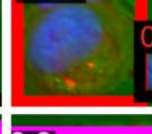

0.9

0.9 - 0.53

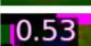

0.
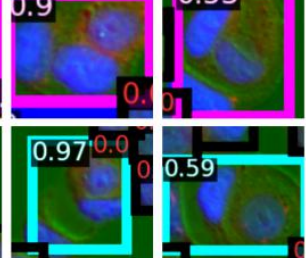

0.59

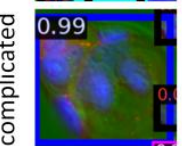

0.99 
bioRxiv preprint doi: https://doi.org/10.1101/2021.02 26.432996; this version posted February 27, 2021. The copyright holder for this preprint (which was not certified by peer review) is the author/funder, who has granted bioRxiv a license to display the preprint in perpetuity. It is made available under aCC-BY-NC-ND 4.0 International license.

\section{Figure 6}

a

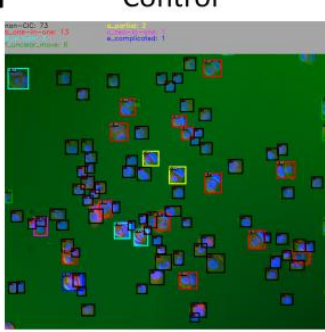

b
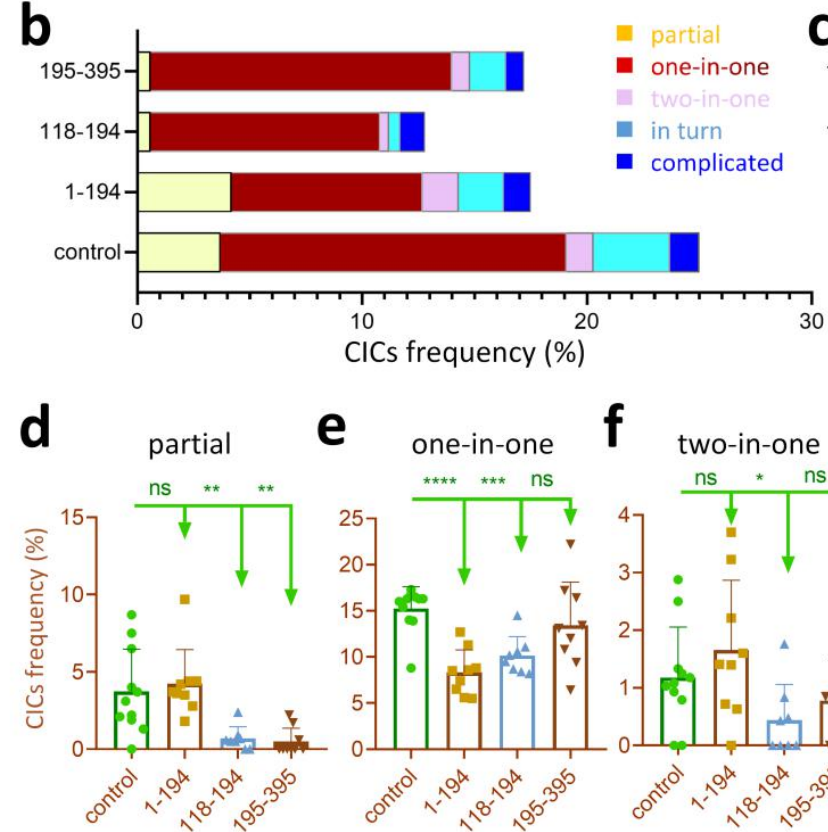

(1-194)

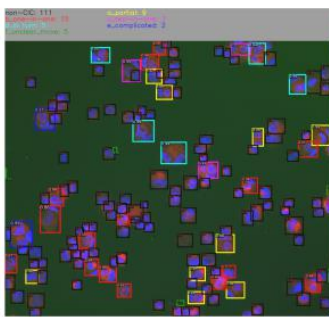

e one-in-one $\boldsymbol{f}$ two-in-one

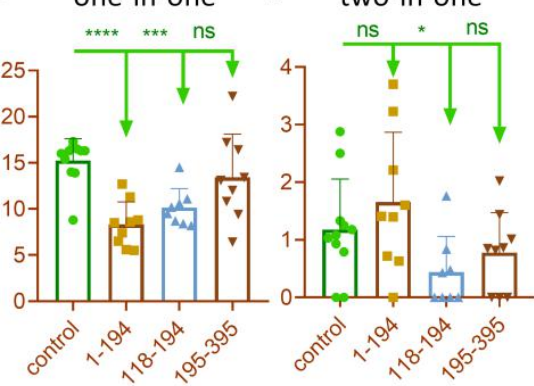

(118-194)

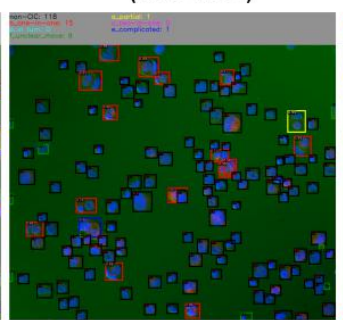

(195-395)

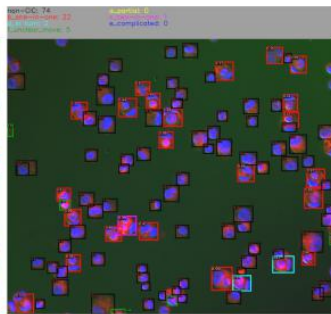

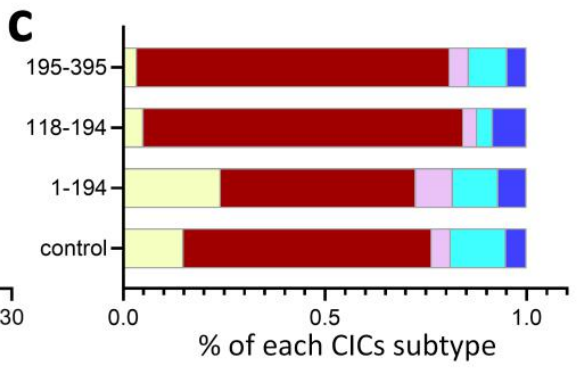

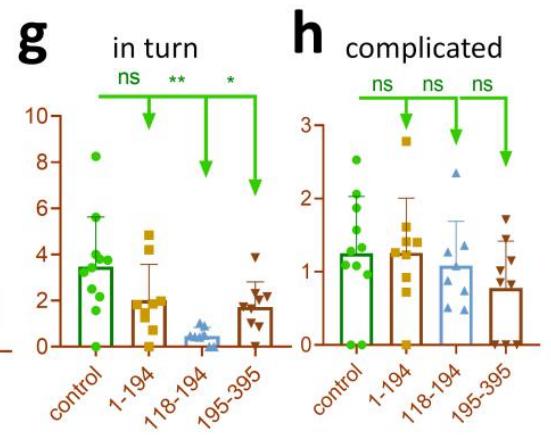


bioRxiv preprint doi: https://doi org/10.1101/2021.02 26.432996; this version posted February 27, 2021. The copyright holder for this preprint (which was not certified by peer review) is the author/funder, who has granted bioRxiv a license to display the preprint in perpetuity. It is made available under aCC-BY-NC-ND 4.0 International license.

\section{Figure S1}

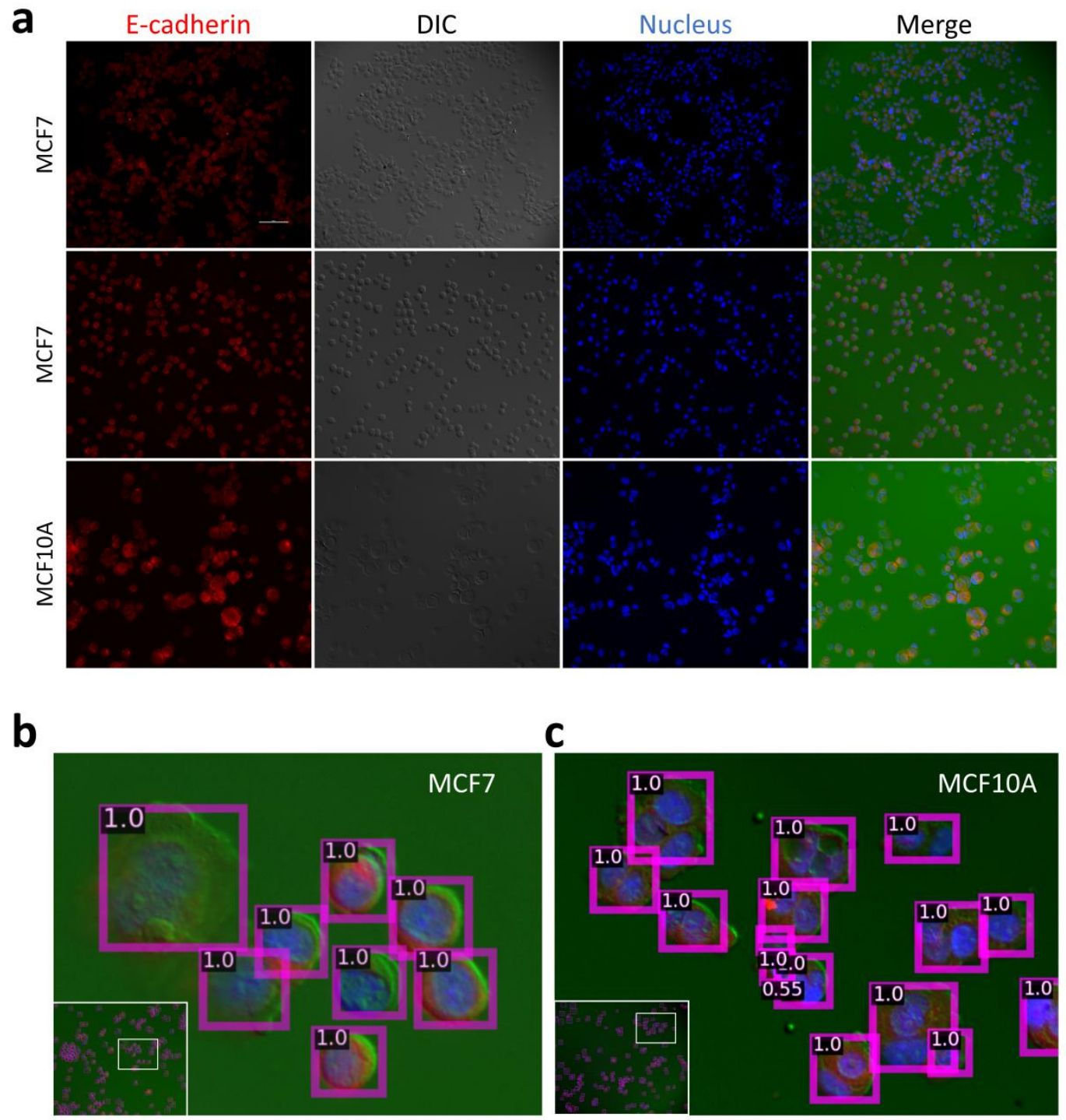


bioRxiv preprint doi: https://doi.org/10.1101/2021.02 26.432996; this version posted February 27, 2021. The copyright holder for this preprint (which was not certified by peer review) is the author/funder, who has granted bioRxiv a license to display the preprint in perpetuity. It is made available under aCC-BY-NC-ND 4.0 International license.

\section{Figure S2}

a
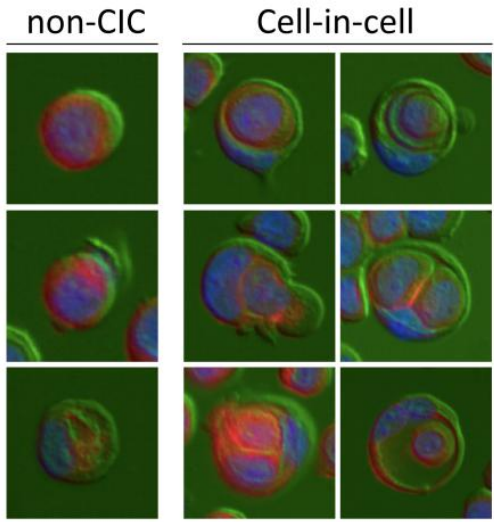

C

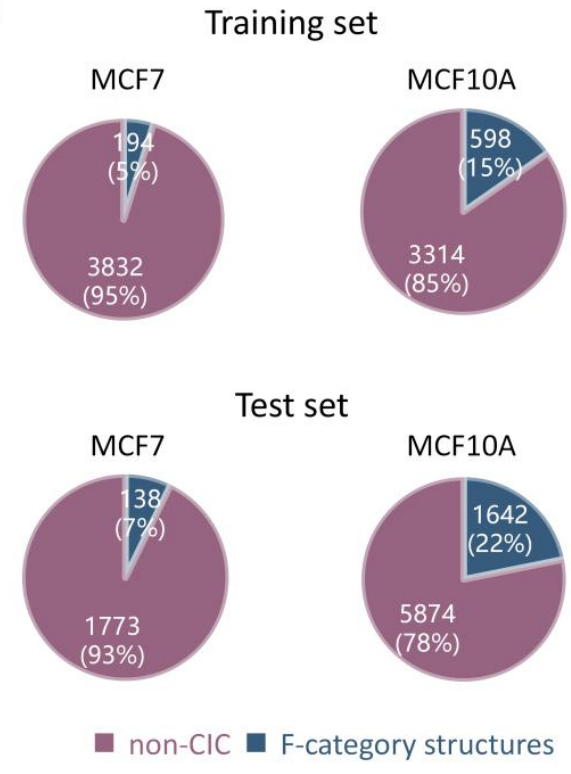

b

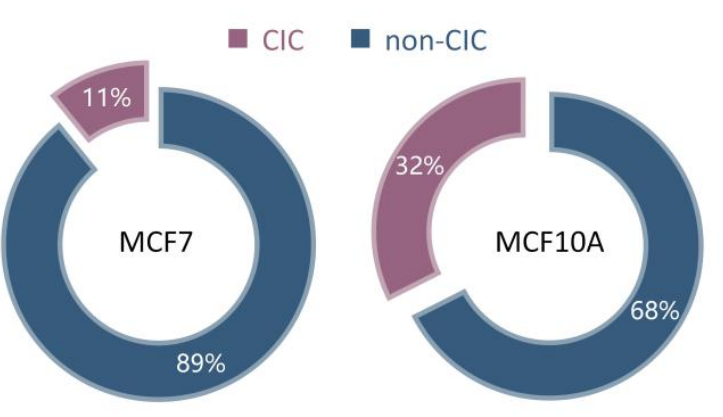

Training set

d

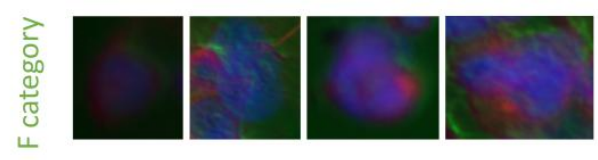

e

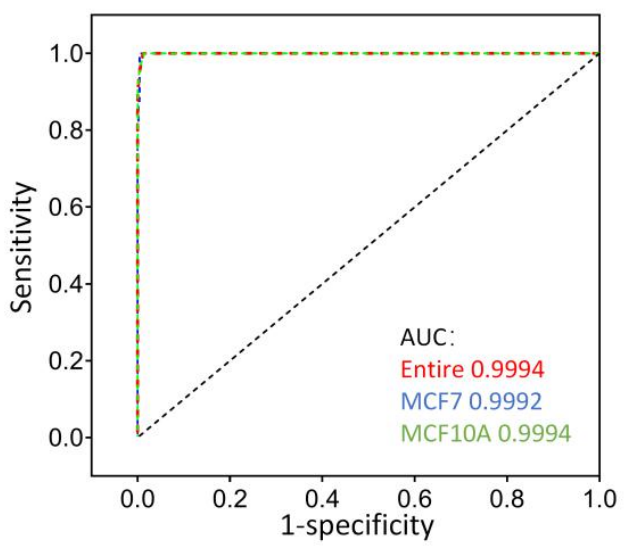

